\title{
Estabilidade Visual na Retinopatia Diabética Tratada por Panfotocoagulação com Laser
}

\begin{abstract}
RESUMO
Objetivo: Avaliar o efeito da "laserterapia" na acuidade visual em portadores de retinopatia diabética avançada e sem queixas visuais. Métodos: Desenvolveu-se estudo observacional descritivo em portadores de retinopatia diabética avançada e sem queixas visuais, submetidos a panfotocoagulação retiniana com laser de argônio. Foi realizado exame oftalmológico completo. Entre o $3^{\circ}$ e o $5^{\circ}$ meses do tratamento, foi repetido o exame inicial. Para comparação da acuidade visual, entre período pré e pós-tratamento, foi utilizado o teste nãoparamétrico de Wilcoxon. Resultados: A amostra foi constituída por 44 pacientes, totalizando 67 olhos, sendo que $37(55,2 \%)$ apresentavam retinopatia diabética proliferativa e $30(44,8 \%)$ não proliferativa grave ou muito grave. A acuidade visual pré-tratamento variou de 0,5 a 1,0 (média de $0,83 \pm 0,17$ ). Não houve alteração estatisticamente significante na acuidade visual $(p=0,057)$ pré e pós-tratamento no período estudado. Conclusão: Houve estabilidade visual pós-tratamento com a panfotocoagulação, o que enfatiza a necessidade de intervenções em fases mais precoces da retinopatia diabética para preservar a função visual. (Arq Bras Endocrinol Metab 2007;51/4:575-580)
\end{abstract}

Descritores: Retinopatia diabética/terapia; Angiopatias diabéticas; Diabetes mellitus/complicações; Retinopatia diabética/prevenção e controle; Retinopatia diabética/diagnóstico; Saúde pública

\begin{abstract}
Visual Stability in Diabetic Retinopathy Treated by Panretinal Laser Photocoagulation.

Purpose: To evaluate the effect of laser treatment in the visual acuity caused by advanced diabetic retinopathy without visual complaints. Methods: A descriptive observational study was developed in advanced diabetic retinopathy patients not presenting visual complaints. The patients were submitted to argon laser panretinal photocoagulation after ophthalmological examination as well as retinography and retinal angiofluoresceinography. The same initial exams were repeated between third and fifth months after the treatment. The non-parametric Wilcoxon test was employed for statistical evaluation between the visual acuities before and after the treatment. Results: The sample involved 67 eyes of 44 patients. Of these 67 eyes, $37(55.2 \%)$ presented proliferative diabetic retinopathy and $30(44.8 \%)$ showed severe and severe non proliferative diabetic retinopathy. The visual acuity before the treatment ranged from 0.5 to 1.0 (mean $0.81 \pm 0.16$ ). There was no significant statistical difference between the visual acuity $(p=0.057)$ before and after the treatment during the follow-up period of five months. Conclusion: The visual stability after panretinal argon laser photocoagulation indicated the need of this treatment in early phases of diabetic retinopathy in order to preserve the visual function.
\end{abstract} (Arq Bras Endocrinol Metab 2007;51/4:575-580)

Keywords: Diabetic retinopathy/therapy; Diabetic angiopathies; Diabetes mellitus/complications; Diabetic retinopathy/prevention \& control; Diabetic retinopathy/diagnosis; Public health artigo original

\author{
OTACÍLIO de O. MAIA JÚNIOR \\ WALTER YUKIHIKO TAKAHASHI \\ MARIA TERESA B.C. BONANOMI \\ ROBERTA FERRARI MARBACK \\ NEWTON KARA-JOSÉ
}

Setor de Retina e Vítreo, Departamento de Oftalmologia do Hospital das Clínicas da Faculdade de Medicina da Universidade de São Paulo (HC-FMUSP), SP.

Recebido em 06/10/05 Revisado em 04/05/06 e 24/07/06 Aceito em 04/12/06 
A

RETINOPATIA É UMA DAS COMPLICAÇÕES mais temidas do diabetes mellitus (DM), devido à incapacidade funcional que ela provoca. Está diretamente associada à falta de controle metabólico (1) e apresenta fatores agravantes como a nefropatia e a hipertensão arterial $(2,3)$. Existem duas situações diferentes em que o paciente diabético pode ter comprometimento da visão: presença de edema macular clinicamente significativo ou complicações da retinopatia diabética proliferativa que, habitualmente, são associadas com perda visual severa, impossibilitando o indivíduo de realizar tarefas simples do cotidiano.

Não existe cura para a retinopatia diabética (RD). Os estudos multicêntricos realizados (DRS Diabetes Retinopathy Study; ETDRS - Early Treatment Diabetic Retinopatby Study; DRVS - Diabetic Retinopathy Vitrectomy Study; DCCT - Diabetes Control and Complications Study; WESRD - Wisconsin Epidemiologic Study of Diabetic Retinopathy; UKPDS United Kingdom Prospective Diabetic Study) estabeleceram a importância dos fatores de risco, seguimento e manejo da RD (4). Apesar de a terapêutica farmacológica para controle das complicações estar sendo pesquisada (fatores anti-angiogênicos e anti-proliferativos), uma vez instalada a retinopatia e detectada a potencialidade de perda de visão, a fotocoagulação da retina, através da utilização de laser de vários comprimentos de onda, é o tratamento de escolha, evitando perda visual em casos selecionados e estabilizando a progressão da doença (5).

O ETDRS definiu as estratégias do tratamento da RD de acordo com a sua classificação (6) e padronizou a técnica para aplicação do laser $(7,8)$. Segundo este estudo, o laser focal ou em grade na mácula deve ser aplicado no edema macular clinicamente significativo e no edema difuso, respectivamente; a panfotocoagulação da retina é indicada para $\mathrm{RD}$ não proliferativa muito grave e para $\mathrm{RD}$ proliferativa $(8,9)$. $\mathrm{O}$ ETDRS demonstrou que o tratamento precoce com laser reduz o risco de piora da visão em mais de $50 \%$ (10), apesar da acuidade visual não ser um parâmetro para respectiva indicação $(8,9)$. O laser exerce papel fundamental no tratamento da $\mathrm{RD}$ e visa primordialmente à prevenção da perda visual, não restaurando a visão já perdida. A dificuldade na prevenção da perda funcional é que muitos pacientes são examinados tardiamente, perdendo-se a oportunidade de evitar complicações importantes da RD (11).

Avaliando-se tempo de doença, intervalo entre o diagnóstico de diabetes e primeiro exame oftalmológico e controle glicêmico dos pacientes, o objetivo deste estudo é analisar o efeito da "laserterapia" na acuidade visual em portadores de RD avançada e sem queixas visuais. Este trabalho faz parte de pesquisa mais ampla acerca desta problemática.

\section{MATERIAL E MÉTODOS}

Foram selecionados para este estudo observacional descritivo, 49 (quarenta e nove) pacientes do Departamento de Oftalmologia do Hospital das Clínicas da Faculdade de Medicina da Universidade de São Paulo (HC-FMUSP) portadores de RD, com indicação primária de panfotocoagulação retiniana, conforme critérios do ETDRS. Outros critérios de inclusão foram: indivíduos de ambos os sexos, qualquer idade, sem outro tipo de maculopatia ou queixas de diminuição da acuidade visual. Aqueles que apresentaram complicações da RD (hemorragia vítrea e/ou descolamento de retina) antes de iniciar o tratamento foram excluídos.

Determinou-se tempo de diagnóstico da doença e intervalo de tempo decorrido entre diagnóstico e a primeira avaliação oftalmológica dos pacientes. O controle metabólico foi avaliado apenas pelos níveis da hemoglobina glicada $\left(\mathrm{Hb}_{\mathrm{Alc}}\right)$ (método certificado pelo NGSP - National Glycohemoglobin Standardization Program, valor de referência $=$ $4,1$ a $6,5 \%)$.

Os pacientes foram submetidos ao exame oftalmológico completo, incluindo medida da acuidade visual com melhor correção pela tabela de Snellen e a biomicroscopia da mácula. Os exames complementares realizados foram a retinografia (figuras 1 e 2) e angiofluoresceinografia (figura 3).

A programação das aplicações do laser seguiu padronização recomendada pelo ETDRS (figura 4). Entre o $3^{\circ}$ e o $5^{\circ}$ meses do tratamento, foi medida a acuidade visual dos pacientes. Inicialmente todas as variáveis foram anali-

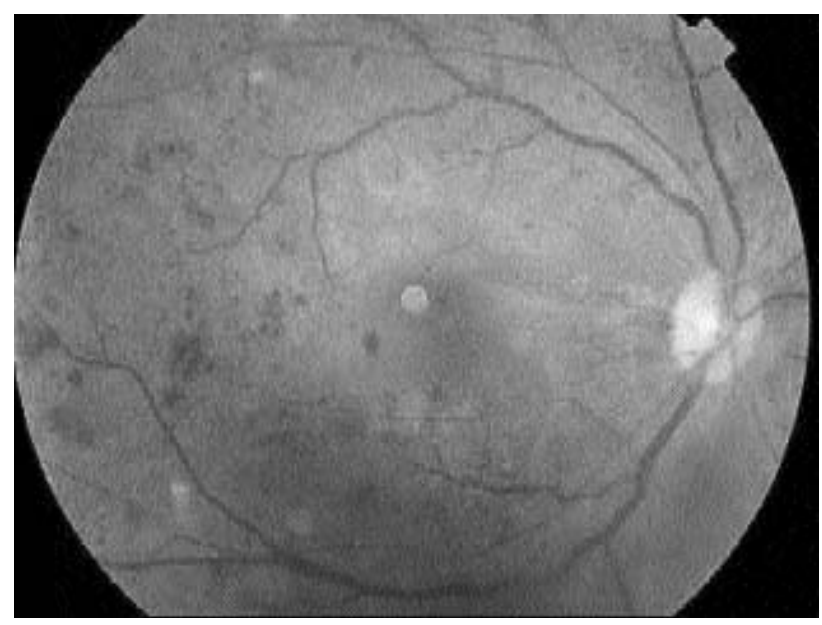

Figura 1. Paciente do sexo feminino, 49 anos, DM há 7 anos e visão de $20 / 20(100 \%)$. Retinografia do $1^{\circ}$ exame mostra hemorragias do tipo dot and blot nos 4 quadrantes e irregularidade da parede venosa, classificando-a como retinopatia diabética não proliferativa muito grave. 


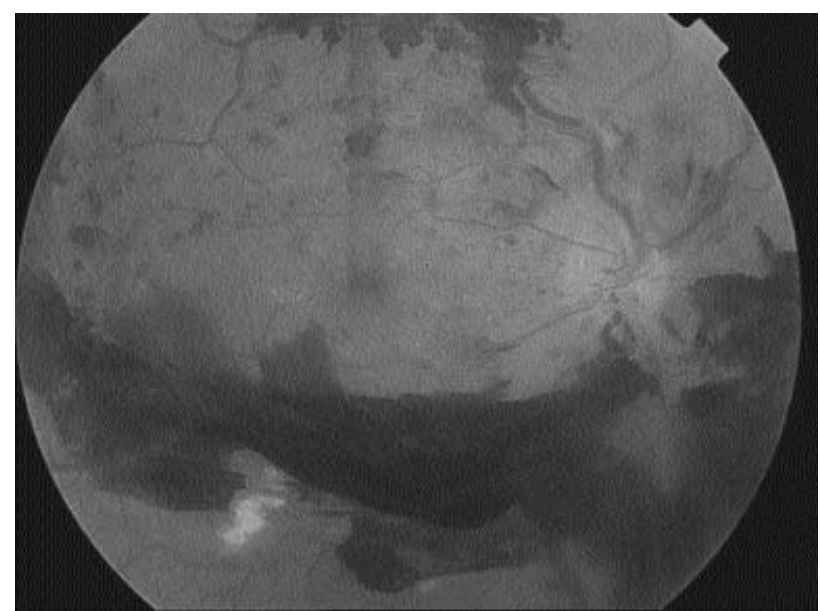

Figura 2. Paciente do sexo masculino, 30 anos, DM há 20 anos e visão de 20/20 (100\%). Retinografia do $1^{\circ}$ exame mostra hemorragias retinianas, manchas algodonosas, raros exsudatos duros e neovasos de papila. A presença de hemorragia vítrea (pré-retiniana) classifica-a como retinopatia diabética proliferativa de alto risco.

sadas descritivamente. Para as variáveis quantitativas, esta análise foi feita por meio da observação dos valores mínimos e máximos e do cálculo de médias, desvios-padrão e medianas. Para as variáveis qualitativas, calcularam-se freqüências absolutas e relativas. Para a comparação entre o período pré e pós-tratamento, foi utilizado o teste não-paramétrico de Wilcoxon. O nível de significância considerado para o teste foi de $5 \%$.

O estudo foi aprovado pela Comissão de Ética para Análise de Projetos e Pesquisa da Diretoria Clínica do HCFMUSP, e todos os pacientes assinaram o termo de consentimento livre e esclarecido.

\section{RESULTADOS}

Dos 49 pacientes selecionados, cinco apresentaram hemorragia vítrea antes de iniciar o tratamento e foram excluídos. Assim, 44 completaram o seguimento, sendo $38(86,3 \%)$ portadores de DM tipo 2 e 6 $(13,7 \%)$ portadores de DM tipo 1 . No grupo de DM do tipo 2, a idade variou entre 40 e 69 anos (média $52,2 \pm 6,8$ anos), com predominância do sexo feminino $(52,3 \%)$. No grupo com DM do tipo 1 , a idade variou entre 24 e 34 anos (média 29,0 $\pm 4,51$ anos) com predominância do sexo feminino $(66,7 \%)$. Os dados relacionados às variáveis quantitativas $\left(\mathrm{Hb}_{\mathrm{Al}}\right.$, tempo de doença e intervalo de tempo para o primeiro exame fundoscópico) estão apresentados na tabela 1 para os pacientes com DM do tipo 2, e na tabela 2 para os pacientes com DM do tipo 1.

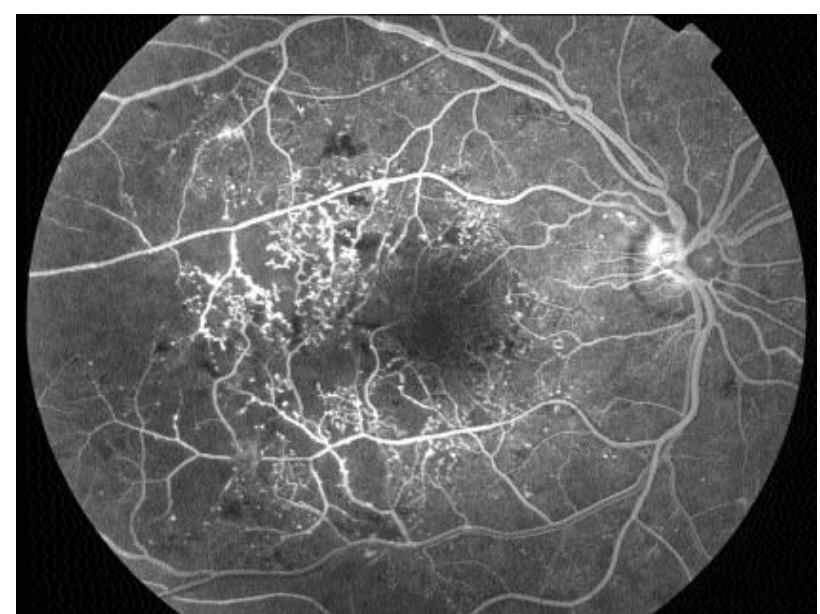

Figura 3. Paciente do sexo masculino, 51 anos, DM há 15 anos e visão de 20/20 (100\%). A angiofluoresceinografia, na $1^{\text {a }}$ consulta oftalmológica, mostra zonas sem perfusão capilar em todo pólo posterior, correspondendo à isquemia retiniana, grande dilatação e aumento da permeabilidade dos capilares ao redor dessas zonas e hiperfluorescência (briIhante) da rede de neovasos na papila.

Os critérios de inclusão foram preenchidos por 67 olhos, $58(86,5 \%)$ entre os pacientes DM tipo 2 e 9 $(13,5 \%)$ entre os pacientes com DM do tipo 1. Quanto ao tipo de retinopatia, $55,2 \%$ apresentavam $\mathrm{RD}$ proliferativa ( 32 olhos de portadores de DM tipo 2 e 5 do tipo 1) e $44,8 \%$ (26 olhos de portadores de DM tipo 2 e 4 do tipo 1) com RD não proliferativa grave ou muito grave.

O tempo decorrido entre o diagnóstico de DM e o primeiro exame de retina sob midríase variou de 3 meses a 18 anos (média de 5,2 \pm 4,81 anos) para os portadores de DM tipo 2, e variou de 7 a 19,5 anos para o tipo 1 (média de $13,2 \pm 5,12$ anos). A hemoglobina glicosilada $\left(\mathrm{Hb}_{\mathrm{Alc}}\right)$ variou de 5,2 a $12,1 \%$ para os DM tipo 2 (média de $8,7 \pm 1,60 \%$ ) e de 7 a $9 \%$ para os tipo 1 (média de $8,6 \pm 0,87 \%$ ).

A acuidade visual pré-tratamento variou de 0,5 a 1,0 (média de $0,83 \pm 0,17$ ). Todos os pacientes foram submetidos a panfotocoagulação retiniana com laser de argônio e a acuidade visual pós-tratamento variou de 0,4 a 1,0 (média de 0,81 $\pm 0,16$ ), após seguimento de 12 a 20 semanas. O teste não-paramétrico de Wilcoxon não mostrou alteração estatisticamente significante da acuidade visual $(\mathrm{p}=0,057)$ pré e pós-tratamento no período estudado (tabela 3 ).

\section{DISCUSSÃO}

O comprometimento significativo da qualidade de vida é freqüentemente relacionado ao diabetes (12), 


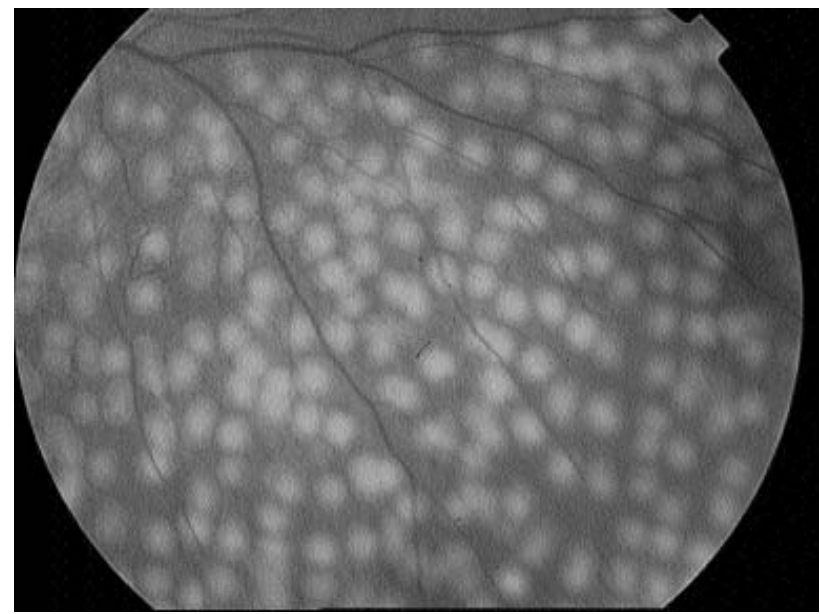

Figura 4. Panfotocoagulação retiniana com laser de argônio (marcas recentes). Nota-se que toda retina isquêmica é fotocoagulada, não apenas o local dos neovasos.

Tabela 1. Dados de portadores de DM tipo 2 com retinopatia diabética avançada e sem queixas visuais. Hospital das Clínicas da Faculdade de Medicina da Universidade de São Paulo - 2005 ( $n=38$ pacientes).

\begin{tabular}{lccccc}
\hline Variável & Média & $\begin{array}{c}\text { Desvio- } \\
\text { padrão }\end{array}$ & Mediana & Máximo & Mínimo \\
Hb1Ac & 8,7 & 1,6 & 8,3 & 12,1 & 5,20 \\
Diagnóstico DM* $^{*}$ & 9,9 & 6,0 & 8,5 & 24,0 & 0,75 \\
1 $^{\text {Fundoscopia** }}$ & 4,6 & 4,3 & 3,0 & 16,0 & 0,09 \\
\hline
\end{tabular}

* tempo de diagnóstico de DM em anos

** anos após o diagnóstico de DM

Tabela 2. Dados de portadores de DM tipo $1 \mathrm{com}$ retinopatia diabética avançada e sem queixas visuais. Hospital das Clínicas da Faculdade de Medicina da Universidade de São Paulo -2005 ( $n=6$ pacientes).

\begin{tabular}{lccccc}
\hline Variável & Média & $\begin{array}{c}\text { Desvio- } \\
\text { padrão }\end{array}$ & Mediana & Máximo & Mínimo \\
& 7,96 & 0,87 & 7,9 & 9 & 7,0 \\
Hb1Ac & 19,30 & 3,81 & 20,0 & 25 & 15,0 \\
Diagnóstico DM* & 6,08 & 6,51 & 4,5 & 18 & 0,5 \\
\hline 1ªundoscopia** & & & &
\end{tabular}

* tempo de diagnóstico de DM em anos

* anos após o diagnóstico de DM

Tabela 3. Acuidade visual, pré e pós-tratamento com laser, em portadores de retinopatia diabética avançada e sem queixas visuais. Hospital das Clínicas da Faculdade de Medicina da Universidade de São Paulo - 2005 ( $n=67$ olhos).

\begin{tabular}{lccccc}
\hline Variável & Média & $\begin{array}{c}\text { Desvio- } \\
\text { padrão }\end{array}$ & Mediana & Máximo & Mínimo \\
\hline $\mathrm{V}^{*}$ pré & 0,83 & 0,18 & 0,9 & 1 & 0,5 \\
$\mathrm{AV}^{*}$ pós & 0,81 & 0,16 & 0,9 & 1 & 0,4 \\
\hline
\end{tabular}

* Acuidade Visual

Nota: Não houve diferença estatisticamente significante na AV entre os momentos avaliados $(p=0,057)$. 
incluindo limitações funcionais, estresse social e financeiro, desconforto emocional e depressão maior (13). A perda da capacidade visual implica no detrimento da qualidade de vida decorrente de restrições ocupacionais, econômicas, sociais e psicológicas (14). Em qualquer estágio da $\mathrm{RD}$ pode ocorrer diminuição importante da visão; no entanto, a cegueira está associada à fase avançada da doença, representada pela RD proliferativa e suas manifestações, como a hemorragia pré-retiniana ou vítrea, proliferação fibrovascular, descolamento tracional de retina, neovasos de íris e glaucoma neovascular (15). No presente estudo fica evidente que, embora os pacientes não apresentaram queixa visual, um número expressivo de olhos $(55,2 \%)$ foi classificado como retinopatia proliferativa e, portanto, sujeito às complicações graves descritas acima. Este fato enfatiza a necessidade de exames periódicos precoces.

Alguns estudos revelam que muitos pacientes com DM são avaliados e tratados de forma errônea e apenas 50\% deles são encaminhados para realização de exame oftalmológico em tempo adequado (16-18). Entretanto, para instituir-se uma efetiva aplicação de laser e prevenir eficazmente a cegueira, o tempo de detecção da RD é crucial (19). Como a diminuição da visão é freqüentemente um sintoma tardio da RD, muitos pacientes permanecem sem diagnóstico, mesmo quando a doença já esteja causando lesões retinianas graves e irreversíveis (11). Dessa forma, perde-se o melhor momento para iniciar o tratamento dos pacientes, ou seja, a fase assintomática, que precede a baixa de visão e outros sintomas oftalmológicos (18), sendo o paciente encaminhado para tratamento apenas em fases avançadas da retinopatia (20). No presente estudo, a média de tempo decorrido entre o diagnóstico de DM e o primeiro exame da retina foi de 5 anos para portadores de DM tipo 2 e de 13 anos para os de tipo 1 , representando um retardo importante desta avaliação pois, apesar de assintomáticos, tiveram indicação de panfotocoagulação retiniana com laser.

A recomendação da Academia Americana de Oftalmologia é que todo paciente com DM tipo 2 seja submetido a exame de retina, sob midríase, no momento do diagnóstico e, do tipo 1, após 5 anos deste. Avaliações posteriores são orientadas anualmente ou mais precocemente, a depender do estágio da RD (21) e do nível de controle glicêmico (22). Uma ação integrada entre o médico que oferece os primeiros cuidados aos pacientes recém diagnosticados e o oftalmologista torna-se imperiosa, podendo reduzir a perda visual (18).
Os resultados obtidos neste trabalho estão em concordância com estudos prévios, que evidenciaram estabilidade da visão após panfotocoagulação com laser de argônio (23). Não foram abordados outros fatores que interferem na $\mathrm{RD}$, como hipertensão arterial sistêmica e nefropatia, que poderiam interferir na visão $(2,3)$. Como estas variáveis não foram controladas no período pré-operatório nem no pós-laser de maneira uniforme para todos os pacientes, acredita-se ser válida a observação de que se consegue manter a visão estável com a panfotocoagulação retiniana, em portadores de RD avançada, independentemente do controle clínico e outros fatores de risco associados. Há uma tendência a indicar esta terapia em fases mais precoces da $\mathrm{RD}$, não aguardando a fase proliferativa da doença, e os pacientes são submetidos ao tratamento muitas vezes sem queixas visuais. Por isso, é de suma importância estudos que avaliem os efeitos da laserterapia na visão, pois a maioria deles aborda as complicações da RD. Uma limitação deste estudo é o tempo de seguimento após tratamento, aparentemente breve, para considerar uma estabilidade definitiva na acuidade visual. Entretanto, como não foram observados sinais de progressão após tratamento, sugere-se uma estabilidade da retinopatia no período avaliado.

Notou-se que, num serviço universitário de referência para tratamento de doenças da retina, há um retardo sistemático dos pacientes com $\mathrm{DM}$ para o $1^{\circ}$ exame oftalmológico. Não houve alteração significativa na acuidade visual com a panfotocoagulação, o que sugere estabilidade da visão pós-tratamento, independentemente do controle clínico. Enfatiza-se a necessidade de exames mais precoces, sendo que maior conscientização dos médicos clínicos e endocrinologistas é necessária para a realização de uma efetiva prevenção da cegueira nos portadores de diabetes.

\section{REFERÊNCIAS}

1. Klein R, Klein BE, Moss SE, Cruickshanks KJ. Relationship of hyperglycemia to the long-term incidence and progression of diabetic retinopathy. Arch Intern Med 1994;154:2169-78.

2. Klein R, Klein BEK, Moss SE, Cruickshanks KJ. The Wisconsin epidemiologic study of diabetic retinopathy (WESDR). XVII. The 14-year incidence and progression of diabetic retinopathy and associated risk factors in type 1 diabetes. Ophthalmology 1998;105:1801-15.

3. Cruickshanks KJ, Ritter LL, Klein R, Moss SE. The association of microalbuminuria with diabetic retinopathy. The Wisconsin Epidemiologic Study of Diabetic Retinopathy. Ophthalmology 1993; $100: 862-7$.

4. Boelter MC, Azevedo MJ, Gross JL, Lavinsky J. Fatores de risco para retinopatia diabética. Arq Bras Oftalmol 2003;66(2):239-47.

5. Ávila M. A retina do século XXI. Arq Bras Oftalmol 2003;66(5):720-30. 
6. Early Treatment Diabetic Retinopathy Study Research Group: Fundus photographic risk factors for progression of diabetic retinopathy. ETDRS report nr. 12. Ophthalmology 1991;98:823-33.

7. Early Treatment Diabetic Retinopathy Study Research Group. Treatment techniques and clinical guidelines for photocoagulation of diabetic macular edema. ETDRS report nr. 2 Ophthalmology 1987;94:761-74.

8. Early Treatment Diabetic Retinopathy Study Research Group. Techniques for scatter and local photocoagulation treatment of diabetic retinopathy. ETDRS report 4. Int Ophthalmol Clin 1987;27:265-72.

9. Early Treatment Diabetic Retinopathy Study Group. Early Photocoagulation for diabetic retinopathy: ETDRS report number 9. Ophthalmology 1991;98(5 suppl):767-85.

10. ETDRS Research Group. Photocoagulation for diabetic macular edema. Early treatment diabetic retinopathy study report number 1. Arch Ophthalmol 1985;103(12):1796-806.

11. Nehemy MB. Retinopatia diabética. Revisão temática. Arq Bras Oftalmol 1998;61(3):366-70.

12. Glasgow RE, Ruggiero L, Eakin EG. Quality of life and associated characteristics in a large national sample of adults with diabetes. Diabetes Care 1997:20:562-7.

13. Anderson RJ, Freedland KE, Clouse RE, Lustman PJ. The prevalence of comorbid depression in adults with diabetes: a meta-analysis. Diabetes Care 2001;24:1069-78.

14. Temporini ER. Promoção de saúde ocular. Arq Bras Oftalmol 1999;62(1):82-4.

15. American Academy of Ophthalmology Basic and Clinical Science Course. Section 12: Retina and vitreous. 1999-2000 [online]. Available <http://149.142.138.19/jseiweb/Education/educ educact basicandclinical.htm>. Accessed in March 2003.

16. Ganley JP. Eradication of blindness secondary to diabetic retinopathy. Epidemiology 1995;2(2):57-8.

17. Sprafka JM, Fritsche TL, Baker R, et al. Prevalence of undiagnosed eye disease in high-risk diabetic individuals. Arch Intern Med 1989;150(4):559-70.
18. Sinclair SH, Delvecchio C. The internist's role in managing diabetic retinopathy: screening for early detection. Cleve Clin J Med 2004;71(2):151-9.

19. González VC, González VME, Martínez DS, Rivera MD, Arredondo PB, Islas AS, et al. A diabetic retinopathy screening program as a strategy for blindness prevention. Arch Med Res 1997;28(1):129-35.

20. Arrata KM, Waszak DMJ, Delai NR, Moreira JR CA. Detecção precoce da retinopatia diabética: análise de 296 pacientes em um hospital universitário. Rev Bras Oftalmol 1996;55(1):63-9.

21. Screening guidelines for diabetic retinopathy. American College of Physicians, American Diabetes Association and American Academy of Ophthalmology. Ann Int Med 1992;116(8):683-5.

22. Sato $Y$, Lee $Z$, Hayashi $Y$. Relationship between mean hemoglobin $\mathrm{HbA} 1 \mathrm{c}$ value and development of proliferative diabetic retinopathy. Jpn J Ophthalmol 2001;45:523-7.

23. Shimura M, Yasuda K, Nakazawa T, Kano T, Ohta S, Tamai M. Quantifying alterations of macular thickness before and after panretinal photocoagulation in patients with severe diabetic retinopathy and good vision. Ophthalmology 2003;110:2386-94.

Endereço para correspondência:

Otacilio de Oliveira Maia Júnior

Rua Adelmário Pinheiro 10

41900-540 Salvador, BA

E-mail: omaiausp@uol.com.br 